\title{
Different deformations of proton and neutron distributions in nuclei
}

\author{
M. Seiwert, P. O. Hess, J. A. Maruhn, and W. Greiner \\ Institut für Theoretische Physik, Johann Wolfgang Goethe Universität, 6000 Frankfurt am Main, Germany \\ (Received 3 November 1980)
}

\begin{abstract}
Different collective deformation coordinates for neutrons and protons are introduced to allow for both stretching and $\gamma$ transitions consistent with experiments. The rotational actinide nuclei ${ }^{234-238} \mathrm{U}$ and ${ }^{232} \mathrm{Th}$ are successfully analyzed in this model.
\end{abstract}

$$
\left[\begin{array}{c}
\text { NUCLEAR STRUCTURE } \\
{ }^{232} \mathrm{Th},{ }^{234-238} \mathrm{U} \text { calculated } B(E 2) \text { values, collective } \\
\text { model. }
\end{array}\right]
$$

In constructing the quadrupole operator of collective models, one of the basic assumptions ${ }^{1,2}$ is the homogeneous charge distribution (HCD) with a uniform ratio of the neutron and proton densities. The qualitative systematic agreement but quantitative disagreement of calculated $B(E 2)$ values with experimental data in the rare earth and actinide nuclei contradicts this assumption. Furthermore, it is a well-known fact that the collective model in the standard formulation yields vanishing probabilities for $M 1$ transitions among any collective nuclear states. ${ }^{3,4}$ This, as well as the lowering of the $g_{R}$ factor from $Z / A$, has been remedied in earlier investigations. ${ }^{3,4}$ Therefore several attempts have been made to generalize collective models in order to describe magnetic nuclear properties consistently. ${ }^{3,4}$ In these works different shapes for the neutron and proton distribution are introduced to allow for an individual behavior of neutron and proton liquid:

$R^{i}(\theta, \varphi, t)=R_{0}\left[1+\sum_{\lambda_{\mu}} \alpha_{\lambda_{\mu}}^{i}(t) Y_{\lambda_{\mu}}^{*}(\theta, \varphi)\right], \quad i=p, n$.

$\alpha_{\mu}^{p}$ and $\alpha_{\mu}^{n}$ are independent degrees of freedom but they are coupled strongly via the symmetry energy which prevents very large differences in the spatial distributions of protons and neutrons. This suggests the introduction of the center of gravity coordinate $\alpha_{\mu}$ and the difference coordinate $\xi_{\mu}$ :

$$
\begin{aligned}
\alpha_{\mu} & =\frac{B_{p} \alpha_{n}^{p}+B_{n} \alpha_{\mu}^{n}}{B_{p}+B_{u}} \cong \frac{N}{A} \alpha_{\mu}^{n}+\frac{Z}{A} \alpha_{\mu}^{p}, \\
\xi_{\mu} & =\alpha_{\mu}^{n}-\alpha_{\mu}^{p},
\end{aligned}
$$

where $B_{p}$ and $B_{n}$ are the proton and neutron mass parameters.

The Hamiltonian is now decomposed as

$$
H=H_{\mathrm{col11}}(\alpha)+H_{\mathrm{so}}(\xi)+H_{I}(\alpha, \xi),
$$

where $H_{\text {coll }}$ describes the common collective motion,

$$
H_{\text {col1 }}=\frac{1}{2} B_{2}[\dot{\alpha} \times \dot{\alpha}]^{[0]}+V(\alpha),
$$

where both the kinetic as well as the potential term are rotational invariants, which may also be expressed in terms of the Euler angles $\theta$, and intrinsic variables $a_{0}, a_{2}$. The principal axes are determined by requiring that the products of inertia of the kinetic energy contained in Eq. (4) vanish. $H_{s o}\left(\xi_{\mu}\right)$ describes the surface oscillations of protons against neutrons. Its typical energy is of the order of giant resonance energy, i.e., $15 \mathrm{MeV}$ (see also estimates by Faessler ${ }^{5}$ ). Because of the small amplitudes involved, a harmonic approximation seems to be appropriate for this proton-neutron-asymmetry degree of freedom.

$$
H_{\text {so }}\left(\xi_{\mu}\right)=\frac{C_{\xi}}{2}[\xi \times \xi]^{[0]}+\frac{B_{\xi}}{2}[\dot{\xi} \times \dot{\xi}]^{[0]} .
$$

The coupling Hamiltonian $H_{I}(\alpha, \xi)$ in lowest order, being also rotationally invariant, must be of the form

$$
\begin{aligned}
H_{I}\left(\alpha_{\mu}, \xi_{\mu}\right)= & \frac{B_{\alpha_{\xi}}}{2}\left\{\left[\dot{\alpha} \times \dot{\xi} f^{0]}+[\dot{\xi} \times \dot{\alpha}]^{[0]}\right\}\right. \\
& +\frac{C_{\alpha_{\xi}}}{2}\left\{[\alpha \times \xi]^{[0]}+[\xi \times \alpha]^{[0]}\right\} .
\end{aligned}
$$

This Hamiltonian has ten degrees of freedom, five for each kind of nucleon. With the assumption of a homogeneous charge distribution $\alpha_{p}=\alpha_{n}=\alpha$ or $\xi=0$, the number of coordinates is reduced to five and the Hamiltonian becomes the well-known $\hat{H}_{\text {col1 }}$ of Eq. (4). It is our goal now to find a more realistic condition to reduce the number of degrees of freedom. We consider the potential energy

$$
\begin{aligned}
V(\alpha, \xi)= & V(\alpha)+\frac{C_{\xi}}{2} \sum_{\mu}\left|\xi_{\mu}\right|^{2} \\
& +\frac{C_{\alpha \zeta}}{2} \sum_{\mu}\left(\alpha_{\mu} \xi_{\mu}^{*}+\alpha_{\mu}^{*} \xi_{\mu}\right) .
\end{aligned}
$$

Determining the $\xi$ value of the potential minimum 
for fixed $\alpha$, we obtain

$$
\left.\frac{\partial V}{\partial \xi_{\mu}}\right|_{\alpha_{\mu}}=C_{\xi} \xi_{\mu}^{*}+C_{\alpha \xi} \alpha_{\mu}^{*} \equiv 0
$$

which leads to

$$
\frac{\xi_{\mu}}{\alpha_{\mu}}=\frac{-C_{\alpha \beta}}{C_{\xi}} \equiv \tan \delta
$$

Considering only excitations near the ground state, the core is forced to oscillate along the direction of the vibration with a constant ratio $\xi / \alpha$. The ratio (8) is constant for the potential of the form (7) because the potential parameters $C_{\alpha \xi}$ and $C_{\xi}$ are constants. For more involved interactions between the $\xi$ and $\alpha$ degrees of freedom this is no longer so. This constancy implies the introduction of new coordinates

$$
\alpha_{\mu}=u_{\mu} \cos \delta, \quad \xi_{\mu}=u_{\mu} \sin \delta .
$$

The Hamiltonian is then reduced to five dimensions,

$$
\begin{aligned}
H(u)= & \left(\frac{1}{2} B_{2} \cos ^{2} \delta+\frac{1}{2} B_{\xi} \sin ^{2} \delta+B_{\alpha \xi} \cos \delta \sin \delta\right) \\
& \times[\dot{u} \times \dot{u}]^{[0]}+\left(\frac{1}{2} C_{\xi} \sin ^{2} \delta+C_{\alpha \xi} \cos \delta \sin \delta\right) \\
& \times[u \times u]^{[0]}+V^{\prime}(u) \\
\equiv & \frac{1}{2} B_{u}[\dot{u} \times \dot{u}]^{[0]}+\bar{V}(u),
\end{aligned}
$$

where we included all the rotationally invariant terms constructed out of $u^{[2]}$ in the potential $\tilde{V}(u)$. The mathematical form of the Hamiltonian corresponds to the usual formulation of collective modes in the coordinates $u^{[2]}$; the energy levels and eigenfunctions will not be modified compared to earlier calculations by this procedure. The quadrupole operator, defined $\mathrm{as}^{2}$

$$
Q_{2 \mu}=\frac{3 Z R_{0}^{2}}{4 \pi}\left\{\alpha_{2 \mu}^{* p}-\frac{10}{\sqrt{70 \pi}}(-1)^{\mu}\left[\alpha^{p} \times \alpha^{p}\right]_{-\mu}^{22}\right\}
$$

depends only on the proton deformation $\alpha_{\mu}^{p}$, which can be written, as follows, in terms of the coordinates $u^{[2]}$ using Eqs. (2) and (9):

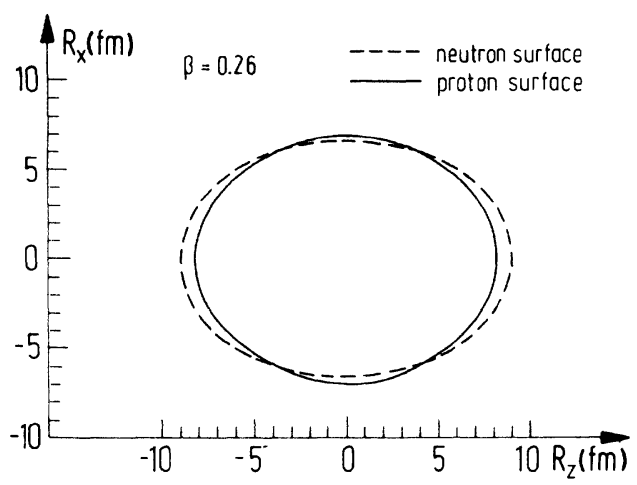

FIG. 1. Proton and neutron distribution for $\beta=0.26$.

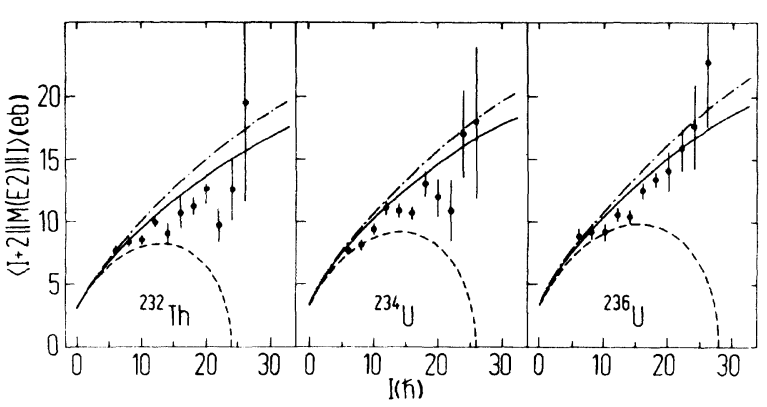

FIG. 2. $M(E 2)$ values plotted as a function of spin $I$ for ${ }^{232} \mathrm{Th},{ }^{234},{ }^{236} \mathrm{U}$. The experimental data are taken from Ref. 6. The predictions of the present work (solid line) are compared to the rotation-vibration model with homogeneous charge distribution (dashed-dotted lines) and the interacting boson approximation in the SU(3) limit (dashed lines).

$$
\alpha_{\mu}^{p}=\alpha_{\mu}-\frac{N}{A} \xi_{\mu}=u_{\mu}\left(\cos \delta-\frac{N}{A} \sin \delta\right) .
$$

Hence, the quadrupole operator depends on the proton-neutron deformation difference $\delta$ :

$$
\begin{aligned}
Q_{2 \mu}=\frac{3 Z R_{0}^{2}}{4 \pi}\left\{u_{\mu}^{*}\left(\cos \delta-\frac{N}{A} \sin \delta\right)\right. & \\
& \left.-\frac{10}{\sqrt{70 \pi}}(-1)^{\mu}[u \times u]_{-\mu}^{[2]}\left(\cos \delta-\frac{N}{A} \sin \delta\right)^{2}\right\} .
\end{aligned}
$$

$\delta=0$ corresponds to the old assumption of equal proton and neutron deformation. As an illustration, the proton and neutron distributions for $\delta=30^{\circ}$ are shown in Fig. 1 . Obviously the neutrons oscillate with a larger amplitude than the protons. Considering rotational excitations the centrifugal stretching is mainly done by the neutrons whereas the proton distribution is more or less constant. This effect is clearly seen in Fig. 2. There, for

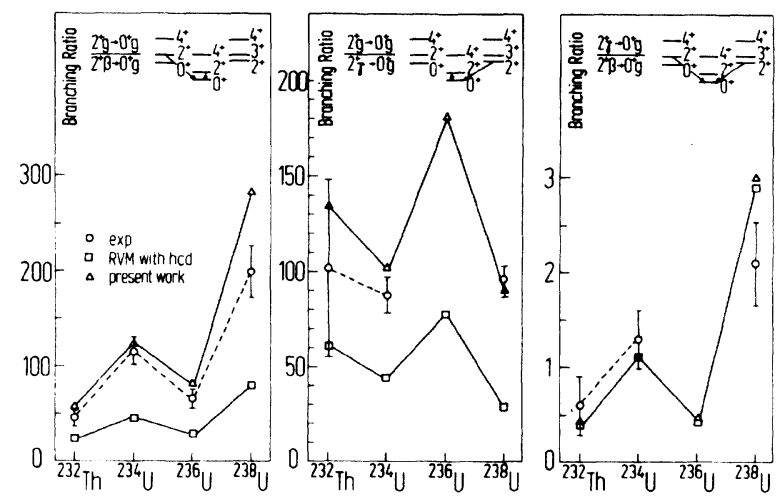

FIG. 3. Different branching ratios for different isotopes. The calculation of the homogeneous charge distribution (HCD) and the present work are compared to experiment (Ref. 9). 
the nucleus ${ }^{232} \mathrm{Th}$ and some $\mathrm{U}$ isotopes, the $M(E 2)$ values are plotted as a function of the spin $I$. The experimental values ${ }^{6,7}$ follow the rigid rotor as if the proton distribution remains constant. In our calculations we use the rotation-vibration model ${ }^{1,2}$ and the reduction of the proton stretching according to the quadrupole operator of Eq. (13) and compare the old and new model predictions with various experiments (Figs. 2 and 3 ).

The predictions ${ }^{6}$ of the interacting boson approximation $^{8}$ seems to be unrealistic for higher spins. The proton-neutron deformation difference $\delta$ in these calculations is fitted to the transition from the $\beta$ to the ground-state band. We obtain values between $23^{\circ}$ and $28^{\circ}$. In Fig. 3 some branching ratios are given. The ratio of an intraband to an interband transition is sensitive to the charge distribution while this is not true for the ratio of two interband or intraband transitions.

Obviously the introduction of different deformations for the proton and neutron distributions im- proves considerably the agreement of the theory with the $B(E 2)$ transitions within the ground-state band at high spins and also with various intrabandinterband branching ratios. In addition, there is the well-known lowering of the collective $g_{R}$ factors from $Z / A$ and also the collective $M 1$ transitions explained by the same idea (mechanism). We may thus conclude that there is considerable evidence for different proton and neutron deformations in nuclei, the neutrons having the larger deformations. This may be due to the smaller pairing force of the neutrons compared to the one of the protons.

The authors would like to thank Professor Th. W. Elze and Dr. H. Ower from the Institut für Kernphysik Frankfurt for illuminating discussions, suggestions, and comments. We also thank Dr. $\mathrm{H}$. Ower for the permission to use some figures of his thesis.
${ }^{1} J$. M. Eisenberg and W. Greiner, Nuclear Theory, 2nd ed. (North-Holland, Amsterdam, 1975), Vol. 1.

${ }^{2}$ A. Faessler, W. Greiner, and R. K. Sheline, Nucl. Phys. 70, 33 (1965).

${ }^{3}$ W. Greiner, Phys. Rev. Lett. 14, 599 (1965).

${ }^{4} \mathrm{~V}$. Maruhn, W. Greiner, and J. A. Maruhn, Phys. Lett. 57B, 109 (1975).
${ }^{5}$ A. Faessler, Nucl. Phys. 85,653 (1966).

${ }^{6} \mathrm{H}$. Ower, thesis, University of Frankfurt/Main, 1980.

${ }^{7} \mathrm{Th}$. W. Elze and H. Ower, private communication.

${ }^{8}$ A. Arima and F. Iachello, Phys. Rev. Lett. 35, 1069 (1975).

${ }^{9}$ Nucl. Data Sheets 20 (1977); 21 (1977). 